DOI: $10.5455 / 2320-1770$. ijrcog20140330

Research Article

\title{
Preterm birth and its outcome
}

\section{Farhin Radhanpuri*, Deepak A. Desai, Jyoti Sharma, Pawanpreet Kaur}

Department of Obstetrics \& Gynecology, SBKS Medical Institute and Research Centre, Pipariya, Vadodara - 391760 , Gujarat, India

Received: 10 January 2014

Accepted: 2 February 2014

*Correspondence:

Dr. Farhin Radhanpuri,

E-mail: farheenradhanpuri@yahoo.com

(C) 2014 Radhanpuri F et al. This is an open-access article distributed under the terms of the Creative Commons Attribution Non-Commercial License, which permits unrestricted non-commercial use, distribution, and reproduction in any medium, provided the original work is properly cited.

\section{ABSTRACT}

Background: Preterm birth is a major challenge in perinatal health care. Most perinatal deaths occur in preterm infants, and preterm birth is an important risk factor for neurological impairment and disability. Preterm birth not only affects infants and their families-providing care for preterm infants, who may spend several months in hospital, has increasing cost implications for health services. This study aims at to study the causes of preterm birth, perinatal outcome, identify the cases which need intervention.

Methods: This is a retrospective study done at the Department of Obstetrics and Gynecology, Dhiraj General Hospital from May 2012 to May 2013. Total 272 preterm deliveries were enrolled for the study.

Results: According to our study, preterm birth percentages $51 \%$ in low socio economic status, $58 \%$ in severe anaemia, $55 \%$ in unregistered, $37 \%$ in h/o previous preterm birth, $41 \%$ b/w $28-32$ wks of gestational age, $46 \%$ b/w 32-36 wks of gestational age.

Conclusions: In our population preterm birth is more common in poor socio economic status, women with anaemia, malnutrition and these factors can be eliminated by proper nutrition and health education by health workers.

Keywords: Preterm birth, Perinatal outcome, Severe anaemia, Proper nutrition, Health education

\section{INTRODUCTION}

"The Obstetrician delivers the baby from mother, the Paediatrician delivers the baby from the Obstetricians."1 Preterm labor is defined by WHO as "The onset of labor prior to the completion of 37 wks of gestation, in pregnancy beyond 20 wks of gestation." 2 Preterm birth remains a leading direct cause of mortality in both developed and developing country settings. The burden of child mortality attributable to preterm birth is reflected by pregnancy outcome. Preterm births are responsible for $75 \%$ of neonatal mortality. Preterm birth is responsible for $50 \%$ of the long term neurologic impairment in children. The incidence of preterm birth has changed little in recent years. Several factors have contributed to the incidence of preterm birth .These factors includes ${ }^{3}$ :

\section{Maternal}

Race, inter-pregnancy interval, pre-pregnancy BMI $=19$, Family history Demographic factors, Psychosocial factors

\section{Pregnancy history}

Previous preterm delivery, Medical disorders: anaemia, hypertension etc.

\section{Pregnancy characteristics}

Infections, cocaine/heroine, cone biopsy/LEEP, abdominal surgery, multiple gestation, periodontal disease, shortened cervix, tobacco, uterine anomalies, vaginal bleeding, hydramnios. 
There is strong association between infection and preterm birth: 4

- Incidence of subclinical histologic chorioamnionitis:

$\begin{array}{ll}50 \% & 24 \text { to } 28 \text { weeks } \\ 10 \% & >37 \text { weeks }\end{array}$

- The smaller the fetus, the more likely the chorioamnion cultures to be positive:

$\begin{array}{ll}80 \% & <1000 \mathrm{~g} \\ 30 \% & >2500 \mathrm{~g}\end{array}$

- Other leading cause in our hospital is cervical insufficiency: Shortened Cervix is less than $3 \mathrm{~cm}$ or a funneling configuration at internal o.s. in $2^{\text {nd }}$ trimester trans-vaginal Ultrasound. Cervical effacement in normal pregnancy at 32 wks. A short cervix confers a higher risk of preterm delivery. ${ }^{5}$ As this study is a retrospective one it was not possible to do these parameters just to tell about commonest causes seen in our hospital.

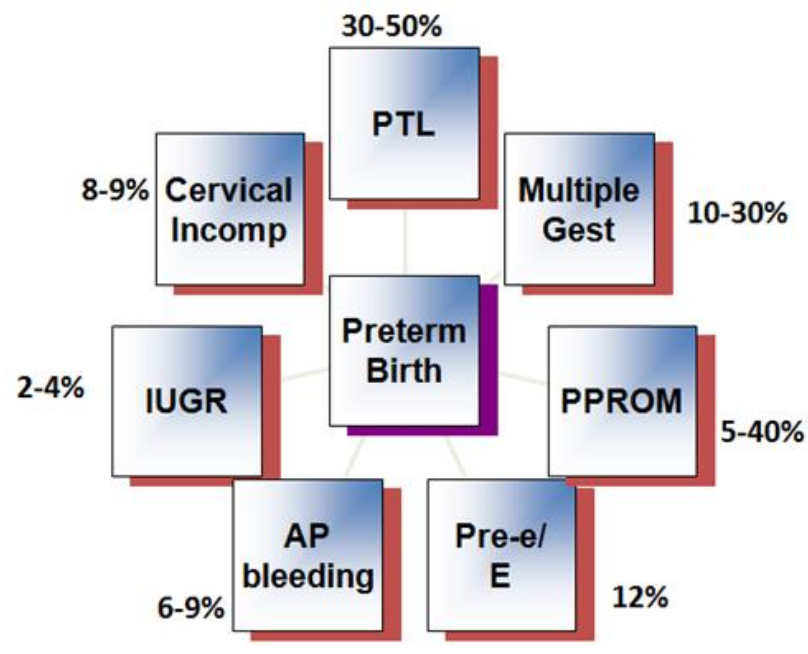

Figure 1: Causes of preterm birth.

In this study we discuss about the most common causes of perinatal mortality and its ratio, trying to find out the causes of preterm labor and birth according to age, parity, nutritional, socio-economic status of the patient.

\section{Aims of the study}

1. To quantify the causes of preterm birth

2. To identify the cases which need intervention

3. To study the perinatal outcome of preterm birth

\section{METHODS}

This is the retrospective study done at the department of Obstetrics and Gynecology. Dhiraj General Hospital from
May 2012 to May 2013. Total 272 preterm deliveries were enrolled for this study.

\section{Inclusion criteria}

1. <37 weeks and >20 weeks of gestational age

2. Singleton pregnancy

3. All booked and un-booked patients

4. Either vaginal preterm delivery or instrumental delivery or c.s.

\section{Exclusion criteria}

1. Pregnancy beyond 37 weeks

2. Multifoetal gestation

3. mal-presentation

\section{Study design}

All the eligible cases were studied from previous data, total preterm births were excluded from fullterm births and the incidence of preterm birth according to maternal age, parity, gestational age at that time, nutritional status of mother, haemoglobin level of mother, perinatal outcome and mortality ratio and common causes of neonatal mortality and morbidity in preterm birth in both booked and un-booked cases are discussed in this study.

\section{RESULTS}

The figure 2 shows total full-term and preterm deliveries from which $84 \%$ preterm normal deliveries, $6 \%$ instrumental preterm deliveries, $10 \%$ c.s.

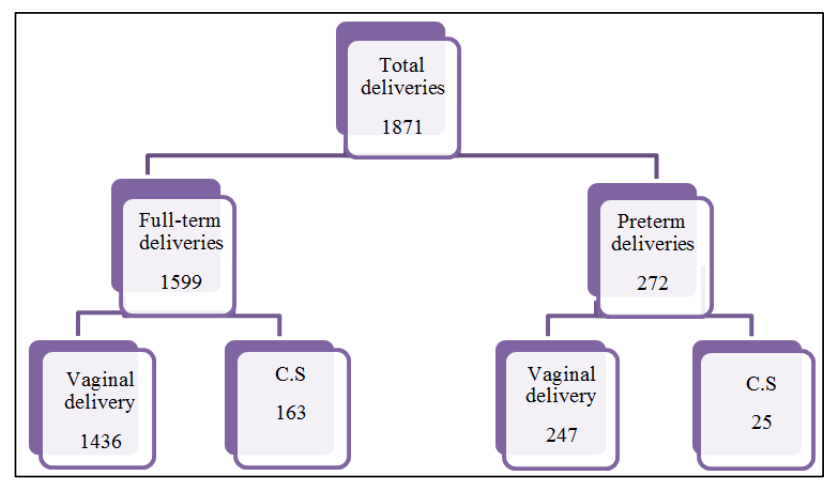

Figure 2: Total full-term and preterm deliveries.

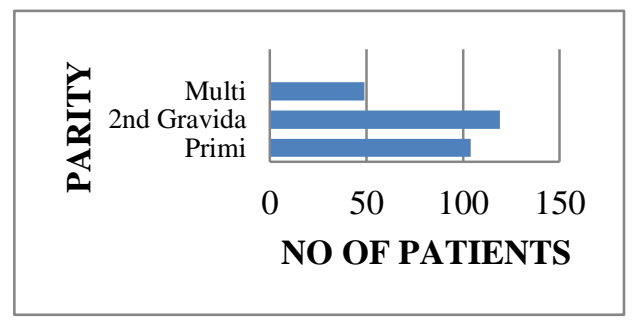

Figure 3: Distribution of preterm birth according to parity. 
The figure 3 shows distribution of preterm birth according to parity. Out of total, 272 pre-term births. The figure 4 shows h/o previous preterm birth according to parity. It shows history of previous preterm birth is the important risk factor for preterm birth.

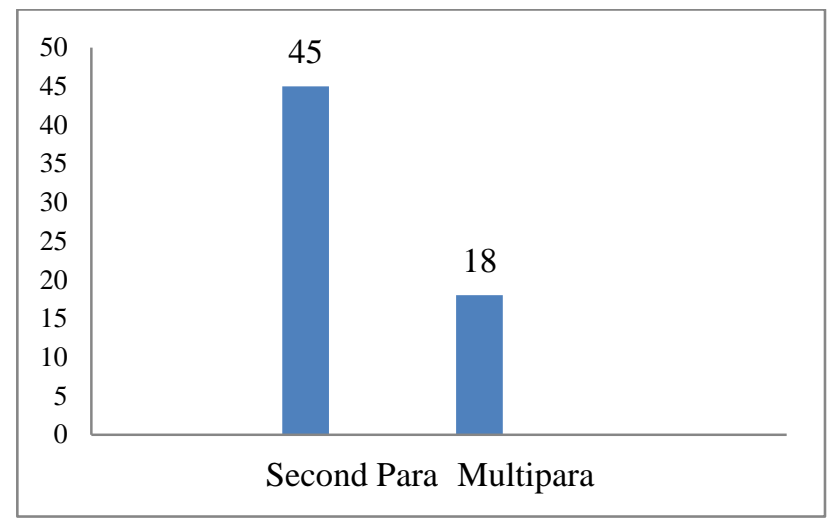

Figure 4: H/o previous preterm birth according to parity.

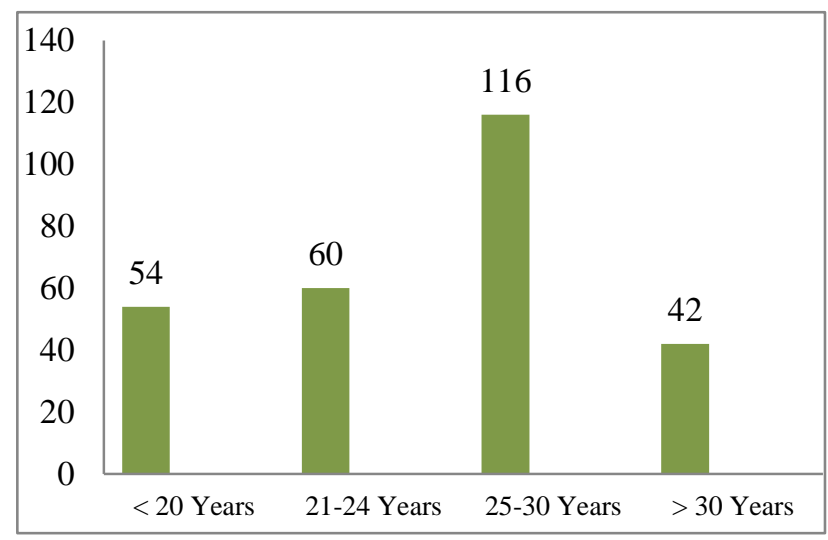

Figure 5: Preterm birth distribution according to maternal age.

The figure 5 shows preterm birth distribution according to maternal age and figure 6 shows h/o previous preterm birth according to age, which shows preterm birth is common with increasing age.

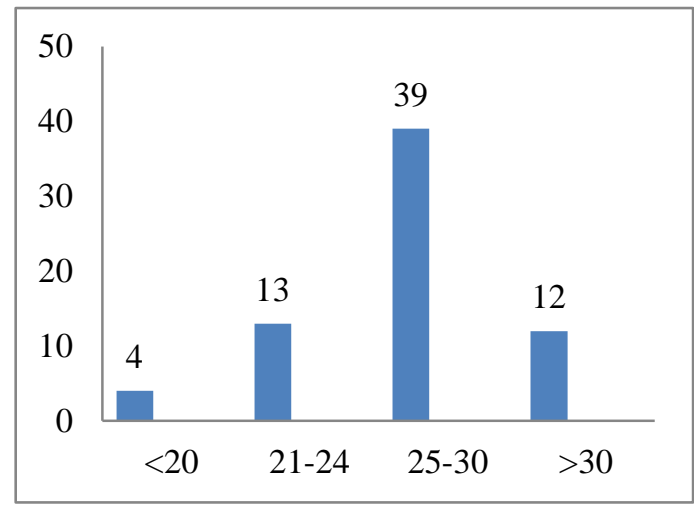

Figure 6: H/o previous preterm birth according to age.

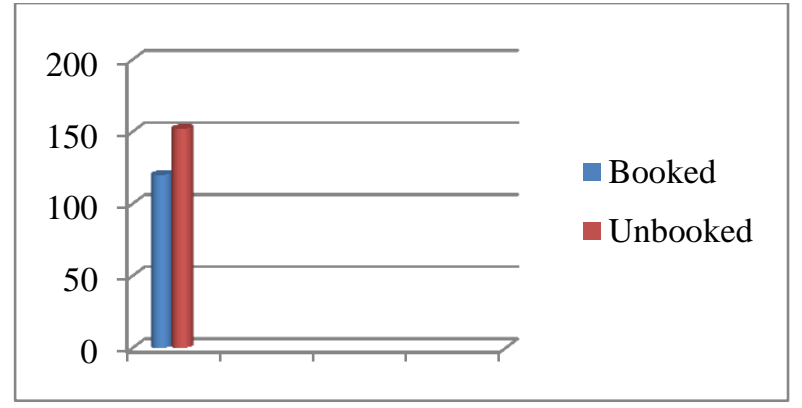

Figure 7: Booked and un-booked cases.

Figure 7 shows booked and un-booked cases out of 272 cases, in which un-booked cases were more.

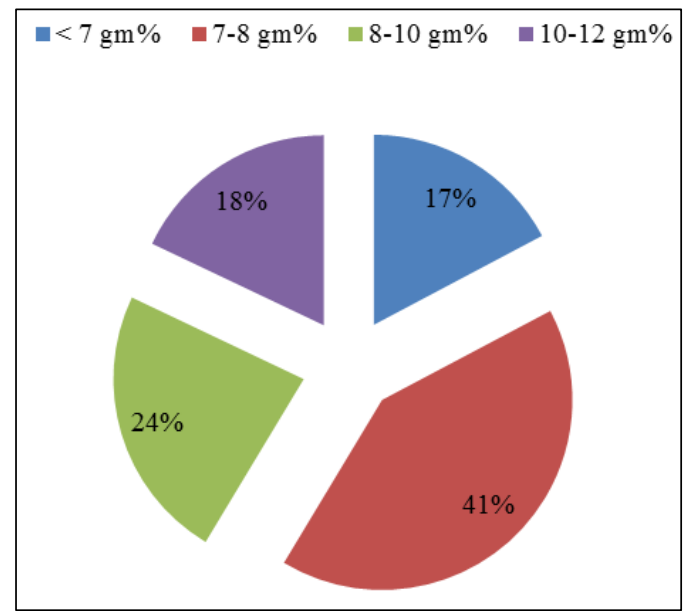

Figure 8: Preterm birth distribution according To anaemic condition of mother.

Figure 8 shows anaemia is the most common risk factor for preterm labor.

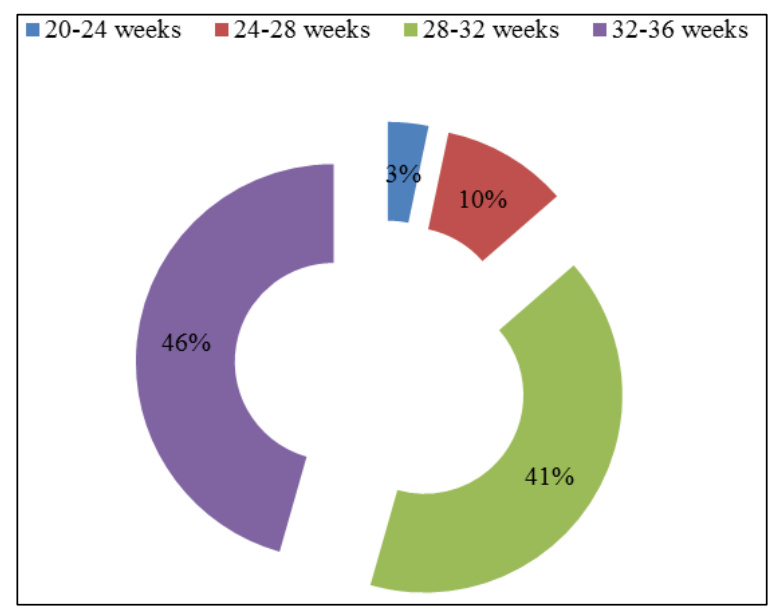

Figure 9: Gestational age at the time of delivery and distribution of preterm birth.

Figure 9 shows gestational age at the time of delivery which shows most of the preterm deliveries occurs at 2836 wks of gestational age. 


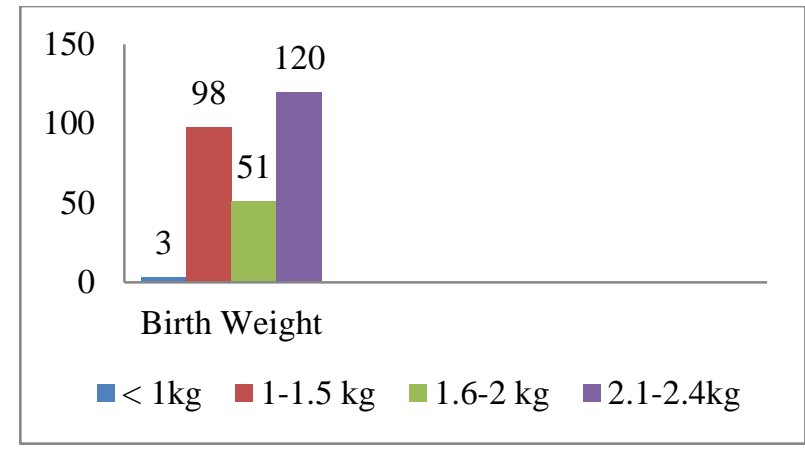

Figure 10: Foetal weight distribution in preterm deliveries.

Figure 10 shows foetal weight distribution in preterm deliveries.

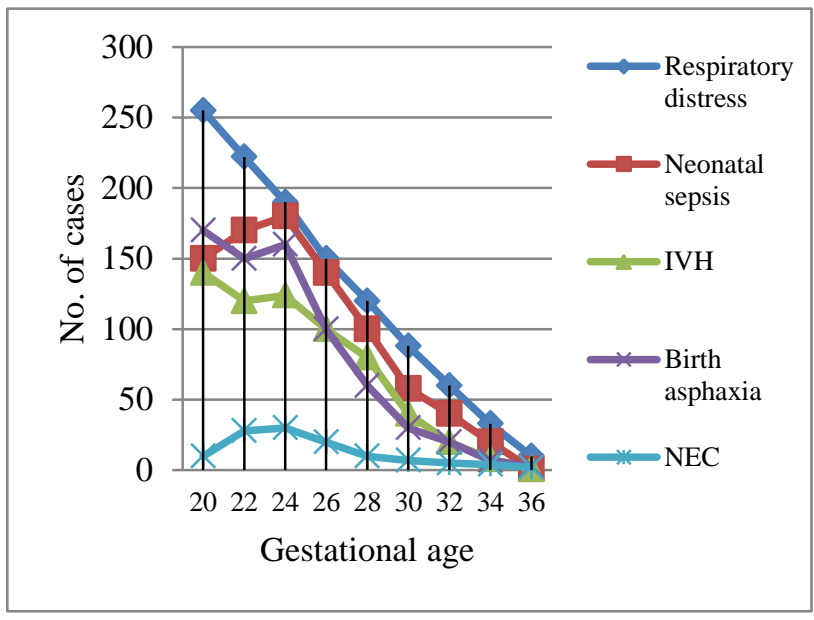

Figure 11: Common causes of neonatal mortality and morbidity in preterm birth seen during this study in our hospital.

Figure 11 shows common causes of neonatal mortality and morbidity in preterm birth seen during this study in our hospital.

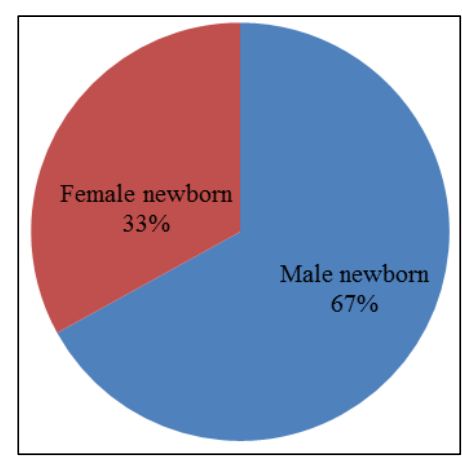

Figure 12: Mortality ratio between male and female new-born due to preterm birth.

Figure 12 shows mortality ratio b/w male and female new-born in which female new-born mortality ratio is very low. May be this is accordance with observation that a female can withstand adversity more effectively than a male baby.

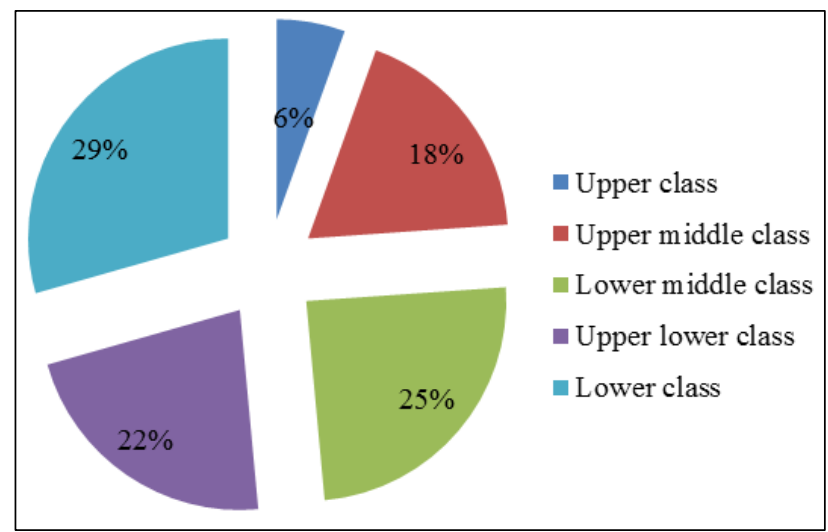

Figure 13: Preterm birth distribution according to socio economic status with kuppuswammy's classification.

Figure 13 shows preterm birth more common in lower socio economic status which affects hygienic condition, nutrition, and psychological status of pregnant women.

\section{DISCUSSION}

According to our study, preterm birth incidence in last year in this hospital was $17 \%$ which is much more than total preterm birth percentage in last year in India which was $7.5 \%$. The study shows anaemia, malnutrition, low socio-economic status, previous preterm birth, increasing birth order are the main contributing factors for preterm birth. Regular antenatal visits, prevention of anaemia, proper nutrition and prior identification of risk factors of preterm labor can reduce the risk of preterm birth. ${ }^{6}$ Though it's very difficult to predict who will deliver prematurely. Preventive strategies revolve around efforts to reduce the background risk factors, screening symptomatic and asymptomatic women for the risk of preterm delivery, and treatment of women with threatened and established preterm labor. Intervention has been aimed at general improvement of nutrition, rest, hydration, and psychological support. According to our study, preterm birth percentages are $51 \%$ in low socio economic status, $58 \%$ in severe anaemia, $55 \%$ in unregistered, $37 \%$ in h/o previous preterm birth, $41 \% \mathrm{~b} / \mathrm{w}$ 28-32 wks of gestational age, $46 \%$ b/w 32-36 wks of gestational age.

\section{CONCLUSIONS}

Inadequate prenatal care is associated with an increased risk of preterm delivery. However this association may be due to other aspects like socioeconomic or psychological factors. $^{7,8}$ Improvement programmes, like increased prenatal visit ,patients education, home visits, nutritional counselling, social and psychological support have effect 
on the rate of preterm deliveries. ${ }^{9,10}$ Though our hospital has facilities of free antenatal visits and routine work up preterm deliveries ratio is high. Screening for risk factors in pregnancy includes monitoring of uterine activity, detection and treatment of infections. Cervical encerclage, tocolysis and progestin therapy, whenever indicated \& possible, may be effective in selected patients in prolonging pregnancy. So regular antenatal visits, supplementation, encouraging proper hygienic conditions will be the most important factors to reduce the percentage of preterm birth.

Funding: No funding sources

Conflict of interest: None declared

Ethical approval: The study was approved by the institutional ethics committee

\section{REFERENCES}

1. Ian Donald's. Hypertensive Disorders. In: Renu Misra, eds. Practical Obstetric Problems. 6th ed. New Delhi: BI Publications; 2012: 397.

2. American College of Obstetrics and Gynaecologists. Preterm labor. Technical bulletin. Washington, D. C.: ACOG; 1995:206.
3. Goffinet F. Primary predictors of preterm labour. $\mathrm{Br}$ J Obstet Gynaecol. 2005 Mar;112(Suppl 1):38-47.

4. Amount RF. Infection in the prediction and antibiotics in the prevention of spontaneous preterm labour and preterm birth. Br J Obstet Gynaecol. 2003 Apr;110(Suppl 20):71-5.

5. Vidaeff AC, Ramin SM. From concept to practice: the recent history of preterm history prevention. Part 1: cervical incompetence. Am J Perinatol. 2006 Jan;23(1):3-13.

6. Simhan HN, et al. Prevention of preterm delivery. $\mathrm{N}$ Engl J Med. 2007 Aug;357(5):477-87.

7. Goffinet F. Primary predictors of preterm labour. $\mathrm{Br}$ J Obstet Gynaecol. 2005 Mar;112(Suppl 1):38-47.

8. David G. Weismiller. Preterm Labor. Amer Family Physician. 1999 Feb;59(3):593-602.

9. Krueger PM, Scholl TO. Adequacy of prenatal care and pregnancy outcome. J Am Osteopath Association. 2000 Aug;100(8):485-92.

10. Fiscella K. Does prenatal care improve birth outcomes? A critical review. Obstet Gynecol. 1995 Mar;85(3):468-79.

DOI: $10.5455 / 2320-1770 . i j \operatorname{cog} 20140330$

Cite this article as: Radhanpuri F, Desai DA, Sharma J, Kaur P. Preterm birth and its outcome. Int J Reprod Contracept Obstet Gynecol 2014;3:153-7. 